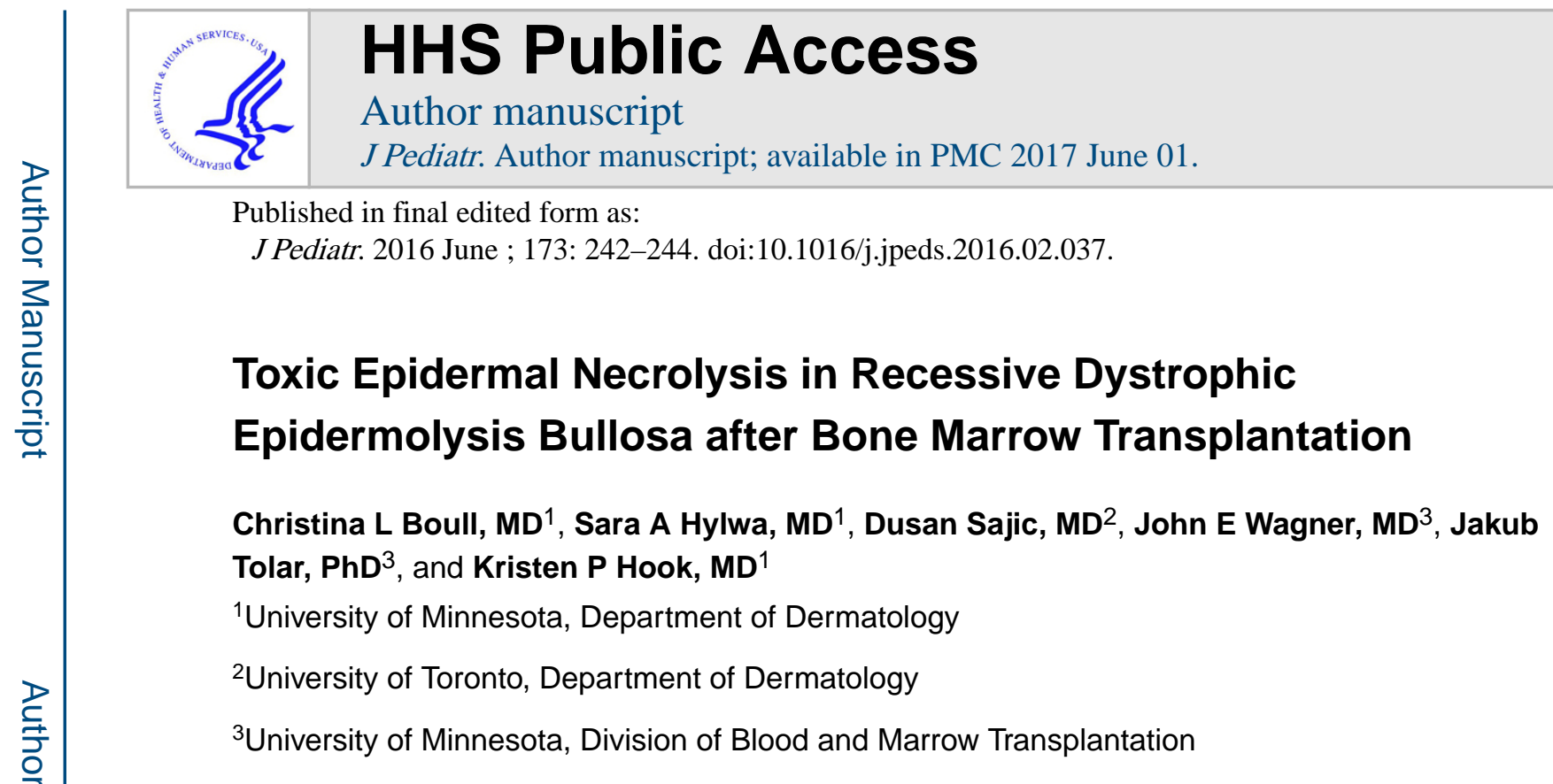

\title{
Report of a Case
}

We present the case of a 3 year-old male with recessive dystrophic epidermolysis bullosa (RDEB) status-post myeloablative allogeneic hematopoietic cell transplant (HCT) and concurrent allogeneic marrow-derived mesenchymal stem cell (MSC) infusion who developed a vesiculobullous eruption and epidermal sloughing.

The patient's course began when he was hospitalized sixteen days post-transplant due to fever in the setting of immunosuppression with cyclosporine and mycophenolate mofetil used as standard graft versus host disease (GvHD) prophylaxis. Vancomycin and ceftazidime were initiated empirically, with transition to cefepime after 24 hours. The patient had known past exposures to all medications. Blood and urine cultures and a chest radiograph failed to reveal an infectious source for his fever.

On hospital day (HD) 3 he developed a pink papular rash that rapidly became vesiculobullous with progressive skin sloughing distinct from his EB lesions. Additional testing included CMV, HHV6, EBV, adenovirus PCR and mycoplasma pneumonia titers and viral and bacterial wound cultures were all negative. His white blood cell count was $1.8 \times 10$ $9 / \mathrm{L}$ with an absolute neutrophil count of $1.4 \times 109 / \mathrm{L}$. Hepatic enzymes were normal. Initial exam by dermatology on HD 11 showed erythema covering $98 \%$ of his body surface area with denuded skin [Fig.1]. No milia or bullae were present and Nikolsky sign was negative. Photophobia, hemorrhagic sloughing of the oral mucosa, and urethral erosions were noted. Toxic epidermal necrolysis (TEN) was confirmed by a biopsy showing full thickness epidermal necrosis with sparse inflammation [Fig.2]. The patient had no other extracutaneous findings suggestive of graft-versus-host disease (GVHD). Engraftment studies of blood showed $40 \%$ donor derived CD15+ leukocytes and 85\% donor derived CD3+ T lymphocytes.

Corresponding Author: Christina L Boull, MD, University of Minnesota, Department of Dermatology, 516 Delaware Street S.E., Mail Code 98, Phillips-Wangensteen Bldg., Suite 4-240, Minneapolis, Minnesota 55455, Tel: (612)625-8625, oehr0005@umn.edu. 
The patient's medication list was reviewed in an attempt to determine an inciting agent. In the month prior to admission he had been exposed to multiple antibiotics including levofloxacin, cefazolin, ceftazidime, cefapime, vancomycin, and prophylactic sulfamethoxazole-trimethoprim. He had been previously treated with all agents without incident. While we suspect that his TEN was related to an antibiotic exposure, we cannot identify the culprit medication with certainty.

At the time of TEN diagnosis all antibiotics were discontinued and intravenous immunoglobulin (IVIG) infusions at a dose of $1 \mathrm{mg} / \mathrm{kg} /$ day were initiated for a total of ten days. Cyclosporine was continued at the same dose while mycophenolate was discontinued, as the patient had completed his transplant protocol determined treatment duration. Wound cares included daily dressing changes with sterile saline irrigation and application of silver sulfadiazine cream or mupirocin ointment to any denuded areas. Wounds were then dressed with non-adherent gauze.

Three days into his IVIG course the patient began to develop diffuse firm, flesh colored, folliculocentric papules [Fig.3]. Due to concern for an atypical presentation of cutaneous GVHD, a biopsy of a papule was obtained, but was consistent only with re-epithelialization. Within three weeks the patient's skin had returned to his baseline with only minimal residual scarring, but he remained hospitalized for an additional two months due to cytopenias and electrolyte disturbances from cutaneous fluid loss.

\section{Discussion}

Pediatric TEN is rare with few cases described in the literature $(1,2)$, usually associated with anti-epileptic medications and antibiotics. While HCT has many well document risks, TEN within the context of HCT has only been described a handful of times $(3,4)$, with roughly half of these cases later re-classified as TEN-like GvHD (Stage IV GvHD) (4). To our knowledge, TEN in a patient with EB has not been described.

Overall, children with TEN fare better than their adult counterparts. In a recent case series of 36 children with TEN treated with IVIG, systemic corticosteroids, or a combination of both all patients survived (1). While adults with TEN in the setting of HCT have risk of mortality approaching $100 \%$ in some case series, it is unclear if this is also true in children (5).

Both TEN and TEN-like GvHD share similar pathogenic mechanisms, and no available laboratory test is able to differentiate them. Furthermore, several authors have reported that GvHD can itself induce the full spectrum of TEN $(4,6,7)$. Clinical observations and the overall course can help differentiate these entities. Patients with TEN-like GvHD usually have systemic manifestations with evidence of serologic liver abnormalities and gastrointestinal disturbances, most commonly diarrhea (4). As our patient had no sustained liver abnormalities and only minimal diarrhea despite extensive epidermal detachment, we favor a diagnosis of TEN over GvHD. His extensive mucous membrane involvement also supports TEN (8). The strongest argument against GvHD in our patient is his rapid reepithelialization without increased immunosuppression. 
We suspect that treatment with cyclosporine and A and MSC infusion favorably altered our patient's clinical course. His concurrent immune suppression with cyclosporine A, previously described as a treatment for TEN, may have decreased his initial disease severity and explain his protracted course. Cyclosporine A may mitigate the inflammatory cascade in TEN by inhibiting IL-2, activation of macrophages, and activation of the Fas ligand-Fas receptor pathways (9-11).

Despite the patient's underlying RDEB and extensive area of skin involvement he healed with minimal scarring. We speculate that MSCs aided in skin repair. MSCs are multipotent cells which can be isolated from a variety of tissue sources. They have the unique ability to migrate to injured tissue and promote repair via anti-inflammatory, anti-microbial, and proangiogenic immune modulating properties (12). In a mouse model, MSC infusion decreased excessive scar formation (13). MSCs were reported to produce rapid wound healing in three patients with drug induced TEN refractory to standard treatments $(14,15)$. There were no adverse events associated with MSC infusion in the TEN patients and only mild transfusion reactions reported in other studies utilizing MSCs.

The patient's recent MSC infusion may also help explain his very unique re-epithelialization pattern. The bulge region of the hair follicle is a known keratinocyte stem cell reservoir. In TEN the follicular unit is relatively spared from necrosis compared to other skin appendageal structures (16-19). Most cases of TEN, however, do not re-epithelialize with the folliculocentric pattern seen here. Perhaps a better explanation would be re-epithelialization arising from the MSC which are also located in the follicular unit. Specifically, the connective tissue sheath surrounding the follicle is the anatomical niche of MSCs (20-24). Both bone marrow MSCs, as infused into our patient, and hair follicle dermal MSCs express the same surface markers (24). In a mouse study infused labeled marrow-derived MSCs localized to hair follicles, sebaceous glands, and blood vessels. Those MSCs that were incorporated into follicles and sebaceous glands were positive for pan-cytokeratin (25). We speculate that in the setting of tissue injury from TEN the patient's infused MSCs homed to their natural locations in the appendageal structures to aid in the re-epithelialization process.

\section{Conclusion}

This article describes a child with DEB who made a complete recovery from TEN. After review of the English-language literature, this is to our knowledge the first reported case of TEN in a patient with EB. Concurrent cyclosporine use and MSC infusion may have contributed to his recovery, despite his recent HCT. We suspect that the patient's MSC infusion may have resulted in the unique folliculocentric pattern of re-epithelialization with minimal scarring, supporting the possible use of MSC infusions as a treatment for TEN.

\section{References}

1. Hamilton GM, Fish J. Pediatric Toxic Epidermal Necrolysis: An Institutional Review of Patients Admitted to an Intensive Care Unit. J Burn Care Res. 2013; 34(6):e351-8. [PubMed: 23816996]

2. Ferrandiz-Pulido C, Garcia-Patos V. A review of causes of Stevens-Johnson syndrome and toxic epidermal necrolysis in children. Arch Dis Child. 2013; 98(12):998-1003. [PubMed: 23873883] 
3. Takeda H, Mitsuhashi Y, Kondo S, et al. Toxic epidermal necrolysis possibly linked to hyperacute graft-versus- host disease after allogeneic bone marrow transplantation. Journal of Dermatology. 1997; 24(10):635-641. [PubMed: 9375462]

4. Villada G, Roujeau JC, Cordonnier C, et al. Toxic epidermal necrolysis after bone marrow transplantation: study of nine cases. J Am Acad Dermatol. 1990; 23(5):870-875. [PubMed: 2254472]

5. Macedo FI, Faris J, Lum LG, et al. Extensive Toxic Epidermal Necrolysis Versus Acute Graft Versus Host Disease After Allogenic Hematopoietic Stem-Cell Transplantation: Challenges in Diagnosis and Management. J Burn Care Res. 2014; 35(6):e431-435. [PubMed: 24476990]

6. Arvidson J, Kildal M, Linde T, et al. Toxic epidermal necrolysis and hemolytic uremic syndrome after allogeneic stem-cell transplantation. Pediatr Transplant. 2007; 11(6):689-93. [PubMed: 17663696]

7. Wu Z, Weimin S. Rash as the first manifestation of acute graft-versus-host disease after orthotopic liver transplantation. European Journal of Dermatology. 2011; 21(6):997. [PubMed: 21856564]

8. Eckardt A, Starke O, Stadler M, et al. Severe oral chronic graft-versus-host disease following allogeneic bone marrow transplantation: highly effective treatment with topical tacrolimus. Oral Oncology. 2004; 40(8):811-814. [PubMed: 15288836]

9. Reese DJ, Henning S, Rockers K, et al. Cyclosporine for SJS/TEN: a case series and review of the literature. Cutis. 2011; 87(1):24-29. [PubMed: 21323097]

10. Paquet P, Jacob E, Damas P, et al. Analytical quantification of the inflammatory cell infiltrate and CD95R expression during treatment of drug-induced toxic epidermal necrolysis. Arch Dermatol Res. 2005; 297(6):266-273. [PubMed: 16249890]

11. Paquet P, Pierard GE. Would cyclosporin A be beneficial to mitigate drug-induced toxic epidermal necrolysis? Dermatol. 1999; 198(2):198-202.

12. Nuschke A. Activity of mesenchymal stem cells in therapies for chronic skin wound healing. Organogenesis. 2014; 10(1):29-37. [PubMed: 24322872]

13. Wu Y, Huang S, Enhe J, et al. Bone marrow-derived mesenchymal stem cell attenuates skin fibrosis development in mice. Int Wound J. 2014; 11(6):701-10. [PubMed: 23409729]

14. Li X, Wang D, Lu Z, et al. Umbilical cord mesenchymal stem cell transplantation in drug-induced Stevens-Johnson syndrome. J Eur Acad Dermatol Venereol. 2013; 27(5):659-661. [PubMed: 22621433]

15. Nakagawa H, Akita S, Fukui M, et al. Human mesenchymal stem cells successfully improve skinsubstitute wound healing. Br J Dermatol. 2005; 153(1):29-36. [PubMed: 16029323]

16. Revuz J, Penso D, Roujeau JC, et al. Toxic epidermal necrolysis: clinical findings and prognostic factors in 87 patients. Arch Dermatol. 1987; 123(9):1160-1165. [PubMed: 3632000]

17. Revuz J, Roujeau JC, Guillaume JC, et al. Treatment of Toxic Epidermal Necrolysis Créteil's Experience. Arch Dermatol. 1987; 123(9):1156-1158. [PubMed: 3631999]

18. French, LE., Prins, C. Toxic epidermal necrolysis. Bolognia, JL.Jorizzo, JL., Schaffer, JV., editors. Elsevier; 2012. p. 323-332.

19. Roujeau JC, Chosidow O, Saiag P, et al. Toxic epidermal necrolysis. J Am Acad Dermatol. 1990; 23(6):1039-1058. [PubMed: 2273103]

20. Sellheyer K, Krahl D. Skin mesenchymal stem cells: Prospects for clinical dermatology. J Am Acad Dermatol. 2010; 63(5):859-865. [PubMed: 20471137]

21. Lako M, Armstrong L, Cairns PM, et al. Hair follicle dermal cells repopulate the mouse hematopoietic system. J Cell Sci. 2002; 115:3967-3974. [PubMed: 12244134]

22. Bajada S, Mazakova I, Richardson JB, et al. Updates on stem cells and their applications in regenerative medicine. J Tissue Eng Regen Med. 2008; 2(4):169-183. [PubMed: 18493906]

23. Kassem M. Mesenchymal stem cells: biological characteristics and potential clinical applications. Cloning Stem Cells. 2004; 6(4):369-374. [PubMed: 15671665]

24. Fritz V, Jorgensen C. Mesenchymal stem cells: an emerging tool for cancer targeting and therapy. Curr Stem Cell Res Ther. 2008; 3(1):32-42. [PubMed: 18220921]

25. Li H, Fu X, et al. Adult bone-marrow-derived mesenchymal stem cells contribute to wound healing of skin appendages. Cell Tissue Res. 2006; 326(3):725-736. [PubMed: 16906419] 


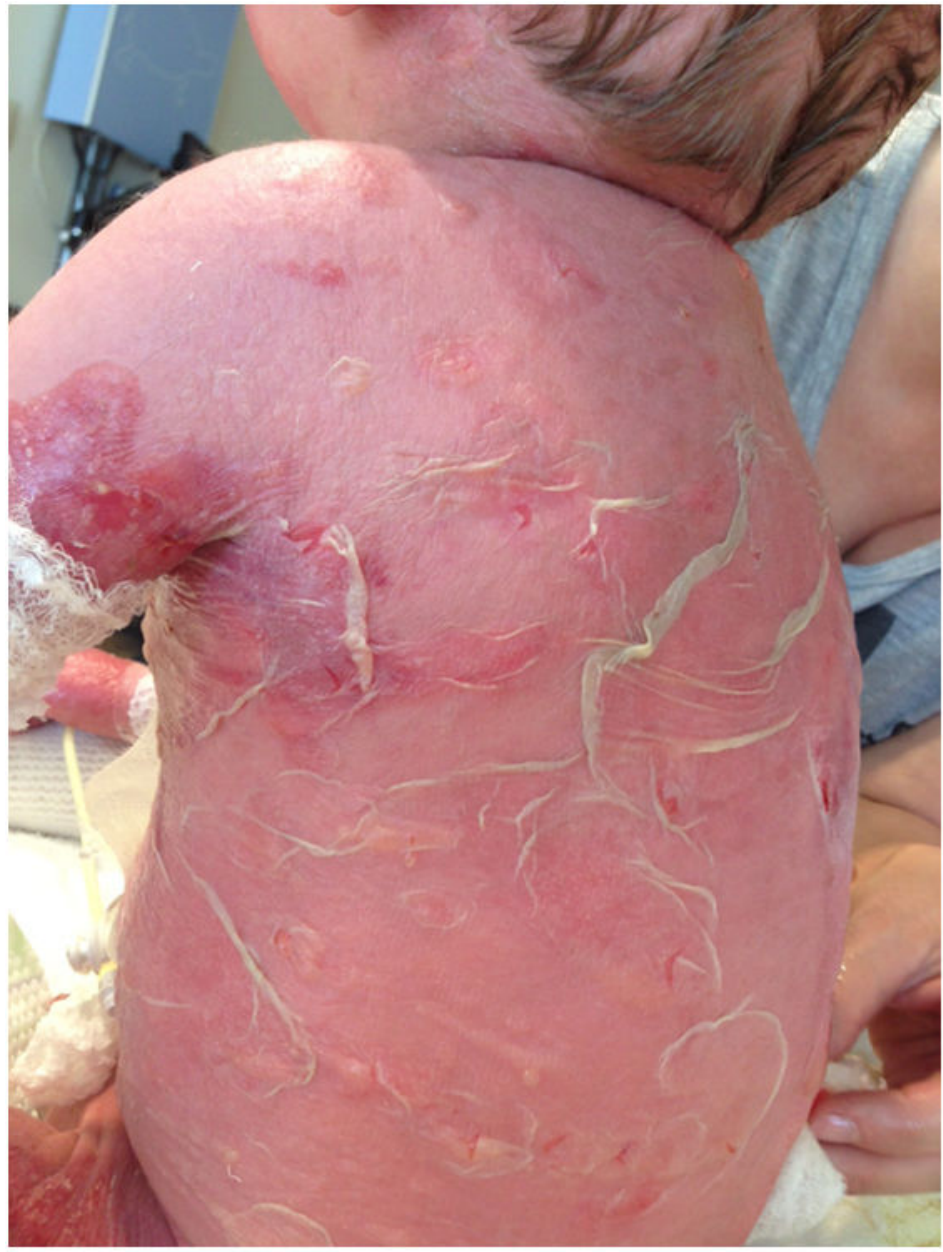

Figure 1.

Patient with full thickness epidermal necrolysis covering $90 \%$ of his body. 

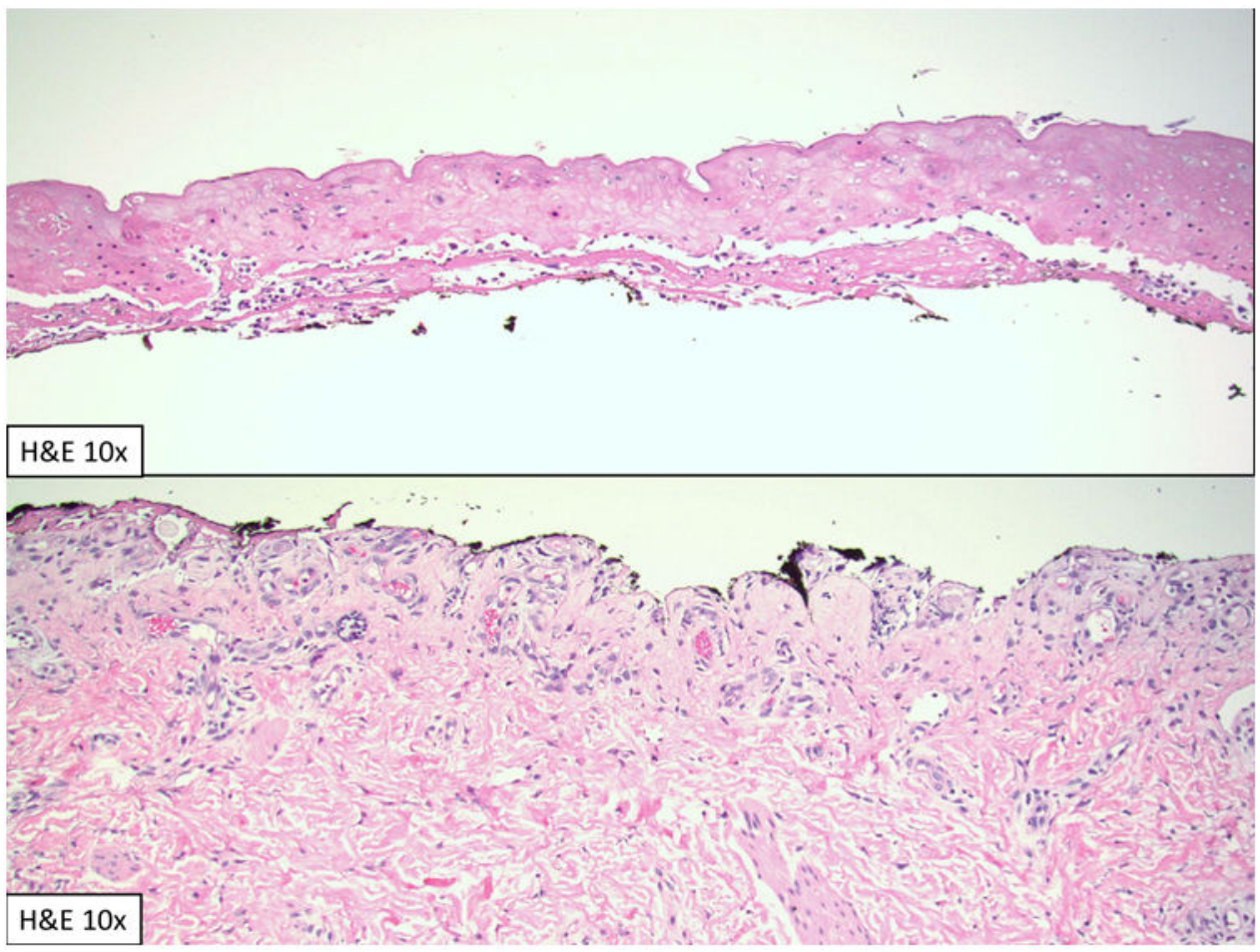

Figure 2.

Biopsy showing full thickness epidermal necrosis with sparse inflammation. 


\section{Figure 3}

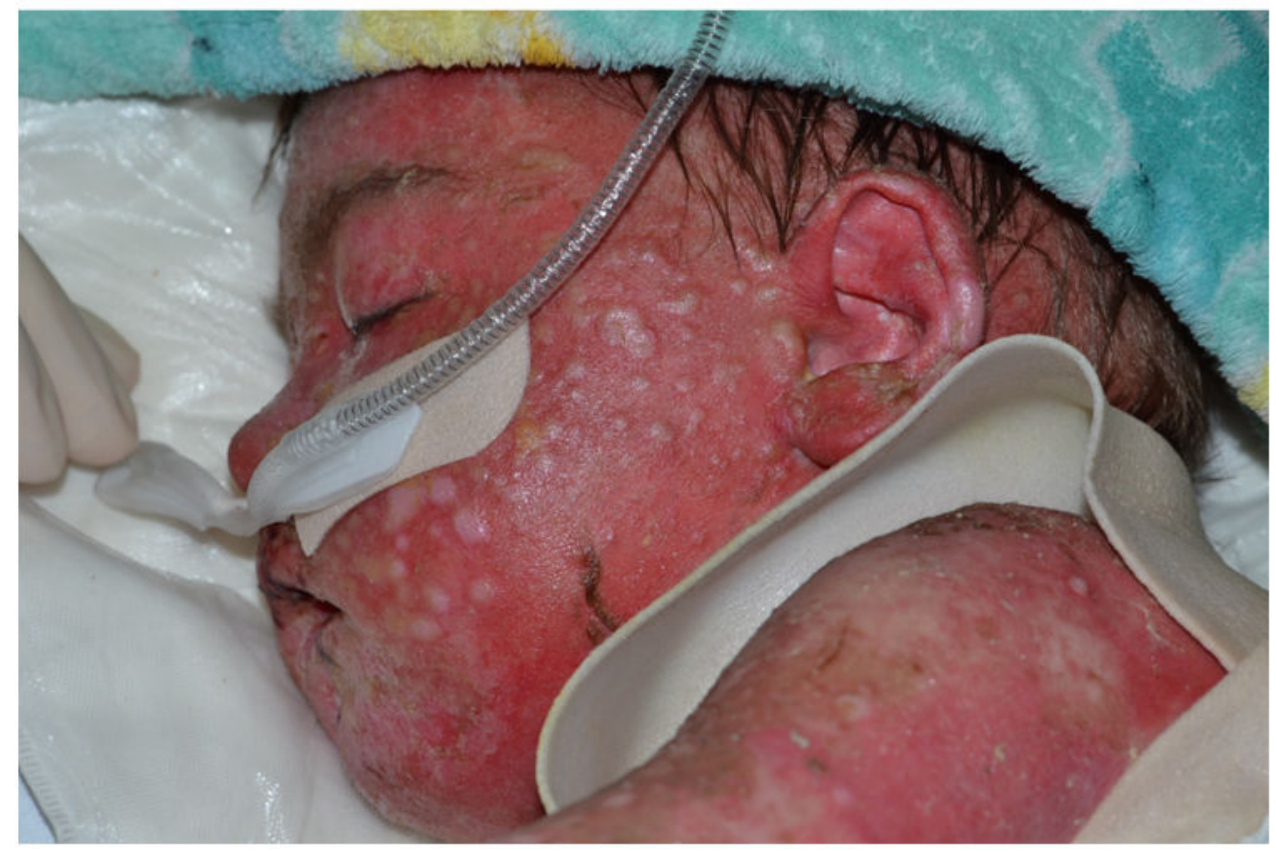

Figure 3.

Patient with diffuse firm, flesh colored, folliculocentric papules. 